\title{
Table-ronde sur Marlowe
}

\section{(2) OpenEdition}

1 Journals

Édition électronique

URL : http://journals.openedition.org/shakespeare/5348

DOI : $10.4000 /$ shakespeare.5348

ISSN : 2271-6424

Éditeur

Société Française Shakespeare

Édition imprimée

Date de publication : 1 novembre 1981

Pagination : 239-243

ISBN : 2-86433-016-4

\section{Référence électronique}

"Table-ronde sur Marlowe », Actes des congrès de la Société française Shakespeare [En ligne], 3| 1981, mis en ligne le 01 novembre 2007, consulté le 23 septembre 2020. URL : http://

journals.openedition.org/shakespeare/5348; DOI : https://doi.org/10.4000/shakespeare.5348 


\title{
THEATRE ET IDEOLOGIES :
} Marlowe, Shakespeare

\author{
DIRECTEUR DE LA PUBLICATION \\ M.T. Jones - Davies
}

JEAN TOUZOT Libraire - Editeur

38 , rue Saint-Sulpice 75278 PARIS CEDEX 061982 
1982 Jean Touzot Libiaire-Editeur, Paris.

Reproduction et traduction mzme partielle interdite.

Tous droits réservts potir lous pays

y compris i'U,R.S.S. et les pays scandinaves

ISBN 2-86433-016-4 


\title{
AVANT-PROPOS
}

L'opposition soulignée par Horace entre éthique et esthétique Aut prodesse volunt aut delectare poetae - que les Elisabéthains aiment à évoquer, pourrait être le lien qui rattache les unes aux autres les communications, rencontres et table ronde de notre troisième congrès (1981) sur le thème : "Théátre et Idéologies: Marlowe, Shakespeare.» Toutefois, le résultat de nos échanges montre un dépassement possible de l'alternative éthique / esthétique, puisqu'il met en valeur l'insertion d'idéologies susceptibles de transformer la fonction même de la littérature.

Les pièces analysées dans le contexte de leur temps révèlent de multiples aspects de la vision du monde et de la place que l'homme $y$ occupe, tels que les projette le théâtre anglais au tournant du XVIe et au début du XVIIe siècle.

Une exploration du langage et de la pensée des deux grands dramaturges aboutit à un commentaire sur les courants philosophiques ou religieux, sur les idées parfois contradictoires et sur la curiosité intellectuelle jamais assouvie qui se font jour à travers la rhétorique de la scène et le jeu dramatique, du Faust de Marlowe à La Tempéte de Shakespeare.

\author{
M.T. jones-Davies
}

Présidente de la Société Française Shakespeare 
SOCIETE FRANCAISE SHAKESPEARE Actes de Congrès.1981

\section{THEATRE ET IDEOLOGIES : Marlowe, Shakespeare}

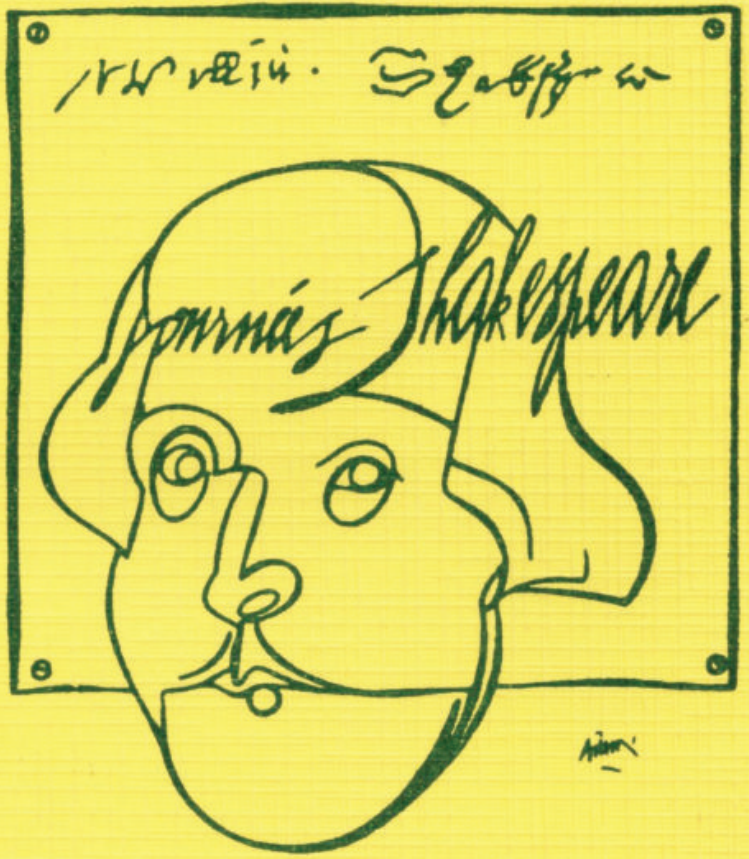

DIRECTEUR DE LA PUBLICATION M.T. Jones - Davies

JEAN TOUZOT Libraire - Editeur 38 , rue Saint-Sulpice 75278 PARIS CEDEX 061982 
Illustration de la couverture : affiche de Valerio Adami, spécialement conçue pour les Journées Shakespeare 1979 au Centre Georges Pompidou 


\section{TABLE DES MATIERES}

M.T. JONES-DAVIES Avant-propos

Théâtre et Idéologies: Marlowe, Shakespeare

S. TRUCHET

The Alchemy of Beauty: aesthetic principles in I Tamburlaine and their relationship to the hermetic ideology.

D. PRUDHOMME

L'idéologie du Juif de Malte et du Marchand de Venise.

D. GOY-BLANQUET

De Hall à Shakespeare : quelques glissements idéologiques opérés par la dramatisation dans Henry $I V$.

M.T. JONES-DAVIES Le Monıde du «si»: Idéologies Incertaines dans Comme il vous Plaira.

G. VENET

Temps et Idéologie : Marlowe, Shakespeare.

J. RICHER

Les Sept Caractères et les Quatre Tempéraments dans Jules César de Shakespeare.

F. LAROQUE

En marge de l'Idéologie : Antimasque et Grotesque dans le Dr Faustus et La Tempête.

A. LECERCLE-SWEET Conscience et Méconnaissance dans Edward The Second de Marlowe.

M.A. CONEJERO Shakespeare's Aesthe tic Scheme. (Extrait).

P.STEWART

Shylock, Shakespeare's Alien.

(Royal Shakespeare Company)

J.RUSSELL BROWN The Romantics' Shakespe are.

R. GILL

The Christian Ideology of Dr Faustus.

J. JACQUOT

Marlowe : de quelques problèmes d'interprétation. 
Rencontres entre Universitaires et Gens de Théâtre

Vendredi 4 décembre 1981
- Stuart Seide :Présentation du

Songe d'une nuit d'Eté. Mise en scène pour le Théâtre de Chaillot janvier 1982

- A propos d'Edouard (II) de

Bernard Turle

Bernard Turle : Métamorphoses de l'auteur et de l'écriture dramatiques. Intervention de Colin Harris (metteur en scène) 
TABLE RONDE SUR MARLOWE 


\section{TABLE RONDE SUR MARLOWE}

A défaut d'une transcription in-extenso de la discussion qui a suivi la lecture de la communication de Professor Roma Gill et l'exposé de J.Jacquot nous reproduisons ici les résumés que les participants nous ont donnés de leurs interventions.

Interventions rassemblées et condensées :

\section{Robert ELLRODT :}

Robert Ellrodt estime que les témoignages concordants sur les opinions de Marlowe doivent contenir une large part de vérité. L'oeuvre manifeste un rationalisme critique à travers le contraste entre la vie des Chrétiens et leurs principes (le point de vue n'est pas celui d'un moraliste). la mise en cause de l'ordre hiérarchique (par Tamburlaine et Gaveston) et de la morale sexuelle (apologie directe ou indirecte de l'homosexualité), l'adoption d'une cosmologie qui n'est pas copernicienne mais qui privilégie l'observation sensible, la critique de notions théologiques telles que la transmission du péché originel (Jew, :I, 2) et la Providence (2 Tamburlaine, II. 3). L'exaltation d'un Dieu déchristianisé, qui est seulement "endless power» ou "revenging wrath", est un appel à une transcendance (homologue de l'ambition humaine au dépassement) mais se nuance d'agnosticisme : The God that sits in Heaven, if any God» (2 Tamburlaine V. 1). On ne peut objecter que le dénouement rétablit l'ordre moral dans Tamburlaine (s'il est frappé pour son impiété envers Mahomet, c'est une parodie du jugement du Dieu chrétien) ni dans Edward II (la note dominante est le défi de Mortimer). La damnation de Faustus est imposée par le sujet et ne peut s'inscrire dans une perspective calviniste (interprétation au premier abord séduisante) sans quelque incohérence. Le trait le plus constant chez Marlowe est l'obsession de la mort et de la souffrance : son rêve - celui de ses héros - rejette moins le Christianisme que la condition humaine.

\section{Sybil TRUCHET :}

Dans Dr. Faustus il faut prendre en considération la nature ambiguë du désespoir accompagnant la damnation 
du héros. D'après Susan Snyder dans son article "The Left Hand of God» (Studies in the Renaissance, 12, 1965) le sentiment éprouvé par Faust peut représenter soit le désespoir salutaire de l'élu ( ce que nous allons retrouver chez. Bunyan), soit le désespoir définitif du réprouvé. Jusqu'à la fin du drame le spectateur s'interroge sur la nature de ce désespoir, car il ne sait pas de quel cóté va pencher la balance.

\section{Danièle PRUDHOMME}

Remarques sur les problèmes religieux dans faust :

$\mathrm{Si}$, dans Le Juif de Malte les trois religions monothéistes - judaïsme, christianisme, Islam - sont stigmatisées, dans Faust Marlowe ne s'en prend qu'au christianisme, mais va plus loin dans ses attaques. Comme dans Le Juif de Malte, il s'en prend au catholicisme (attaque sans grande audace dans l'Angleterre d'après la Réforme) : voir les scènes dans lesquelles les abus de la papauté sont dénoncés, et le pape ridiculisé. Mais Marlowe, à travers Faust, s'en prend aussi à la Réforme. La pièce repose, en fait, sur une double problématique :

1. une problématique catholique : la pièce postule la croyance en la doctrine catholique (ou encore luthérienne) de la grâce. Sans ce postulat, il n'y aurait d'ailleurs plus de pièce. Et si Faust est damné, c'est parce que, jusqu'au bout, il refuse de se repentir et de croire à la possibilité de la rédemption, malgré les exhortations répétées du Bon Ange et du Vieil Homme.

2. une problématique protestante : le protestantisme, et en particulier le calvinisme, n'est pas exempt des attaques de Marlowe. Si Faust refuse de se repentir, son refus n'est pas le produit de la simple ignorance, de l'aveuglement ou de l'orgueil - cette «hubris» si souvent proposée comme explication du comportement des héros marloviens. Faust, docteur en théologie, agit en connaissance de cause. S'il choisit la magie noire et la damnation, c'est par ignorance délibérée, par corruption - ici, par omission - des Ecritures, c'est.à_dire par "perfidie» au sens théologique du terme. Ainsi, lorsqu'il justifie son choix en se référant à une Epítre de Jean, il omet le verset où Jean mentionne la rédemption (I, Jean, i, 9) pour ne conserver que les versets qui mettent l'accent sur le péché 
originel ( v. 8 et 10). Une fois établi que tous les hommes sont pécheurs, la doctrine de la prédestination, qui divise les hommes en «élus» et en "damnés», permet alors à Faust d'inférer que tous les hommes sont damnés, et de justifier son rejet de la théologie au profit de la magie noire (I, 1, 41-47). A mon sens ce passage est, pour Marlowe, une occasion de s'en prendre aux calvinistes, à leur tendance à citer les Ecritures de manière abusive, et de dénoncer les extrêmes auxquels risque de conduire leur obsession du péché et de la damnation.

Les excès du protestantisme sont encore attaqués dans un autre passage de la pièce, où Wagner raille les manières des puritains (precisians) et imite leur discours. (I, 1, 21-26)

\section{Jean-Marie MAGUIN :} de théatre:

1. Caractere subversif de l'utilisation des accessoires

Il me semble que nous avons assez peu parlé du théâtre en tant que lieu physique et jeu. Une note récente de Frances Teague intitulée "Spectacle in Faustus» ( Cahiers Elisabéthains 17. Avril 1980, pp. 83-4) souligne le caractère grossier des tours de magie effectués par Faustus, tours qui nécessitent une participation bienveillante de l'auditoire, à l'inverse de l'authentique et merveilleuse magie qui serait dans la pièce le privilège du diable. De façon générale, j'adhère à cette analyse. Le diable est un bon illusionniste tandis que Faustus se contente de faire des blagues là où il annonce recourir à la magie. En ce sens, les accessoires du théâtre participent à une entreprise subversive tendant à dévaluer le merveilleux en l'assimilant aux techniques de prestidigitation. Marlowe rejoint ici Reginald Scot et sa démystification des illusionnistes et autres escamoteurs, tous faux magiciens, dans The Discoverie of Witchcraft (1584). De la même façon, il est aisé de percevoir la différence de signification entre la bouche d'enfer, élément structurel situé à poste fixe sur la scène médiévale, et sa caricature dans le chaudron du Jew of Malta, enfer artificiel et occasionnel à la mesure d'une vengeance humaine.

2 Sens de la peur du démembrement : (en réponse à une intervention qui voyait là une manifestation de la peur physique)

La peur du démembrement n’est pas nécessairement 
l'expression d'une crainte physique. Elle peut au contraire exprimer une appréhension métaphysique. Selon la théologie de l'époque la dispersion des parties du corps (comme encore aujourd'hui l'incinération pour la théologie catholique) peut faire obstacle à la résurrection de la chair au jour du Jugement et, en conséquence, faire perdre l'éternité. Cette crainte essentielle est, avec d'autres, récupérée et exploitée par la symbolique des tortures et exécutions capitales évoquée par Marienstras. Pour comprendre à quel point, dans ce domaine, chaque détail horrible a un sens on se référera à l'exégèse des châtiments réservés aux accusés de la Conspiration des Poudres faite devant le Parlement par le Procureur Général, Sir Edward Coke.

3. Permanence des débris corporels dans la chambre de Faustus : (en réponse à une intervention voyant là une contradiction avec les termes du pacte selon lequel Faustus se livre au diable «body and soul».)

La permanence des débris corporels découverts par les scholars dans la chambre de Faustus n'est pas contradictoire du pacte aux termes duquel Faustus se livre au diable corps et âme. Le théologien pense en effet qu'en enfer, aussi bien qu'en purgatoire (voir le livre récent de Jacques Le Goff, La naissance du Purgatoire, 1981, p. 16) les âmes sont dotées d'une substance sui generis les habilitant à ressentir des châtiments corporels.

Jacques RAMEL :

"Le Massacre de Paris dont il n'a pas encore été question traite de l'histoire récente, presque de l'actualité, et nous offre en conséquence la vision de la ligne de partage idéologique qui séparait en deux l'Angleterre, l'Europe et méme le monde entier d'un point de vue protestant : aux tenants de la religion réformée, aux Huguenots, à Henri de Navarre, au prince de Condé, à l'Amiral de Coligny et à la reine d'Angleterre s'opposent les catholiques avec, en Angleterre, les séminaristes de Douai/Reims, en France le duc de Guise, Catherine de Médicis et la Ligue, et bien sûr le Pape, l'Espagne et la richesse fabuleuse des Indes. La figure du duc de Guise, avec son envergure héroïque, laisse à penser que, dans ce cadre, dès lors que Marlowe le propagandiste avait rempli son contrat en répétant à 
plusieurs reprises un message idéologique totalement orthodoxe, simple et clair, le dramaturge a repris le dessus; il n'en demeure pas moins que le duc de Guise, comparé à faust ou à Tamerlan, fait figure de petit intrigant préoccupé par ce but matériel qu'est la couronne de France et utilisant pour l'obtenir le machiavélisme le moins glorieux. Un autre problème soulevé par les personnages du Massacre de Paris est la façon dont sont représentés les rois de France Charles IX et Henri III. Marlowe a fait de Charles IX un roi bon et tolérant, mais faible : si les protestants de Paris sont massacrés, c'est malgré lui et non de son fait comme l'écrivaient les polémistes protestants que Marlowe utilisait comme sources. Il y a sans doute là volonté délibérée de ne pas s'en prendre à la famille royale des Valois, volonté qui apparait également dans le portrait du roi Henri III : prince sanguinaire et cynique comme duc d'Anjou, puis jeune roi débauché, il devient au moment de sa mort sage et vertueux, c'est-à -dire ami de la reine d'Angleterre et ennemi du catholicisme. Il aurait en effet été mal venu de la part de l'auteur d'une pièce à la gloire du roi Henri de Navarre de s'en prendre aux Valois, dont Henri de Navarre tirait sa "légitimité.» 